\title{
Identification of blaKPC Gene from Carbapenemase Producing Klebsiella pneumoniae in Thanjavur Medical College
}

\author{
E. Prakash Murthy*, Eunice Swarna Jacob, B. Hari Prasanth and Ayisha
}

Thanjavur Medical College, Tamil Nadu, India

*Corresponding author

A B S T R A C T

\section{Keywords \\ Carbapenemase, Klebsiella pneumonia, Thanjavur Medical College \\ Article Info \\ Accepted: \\ 20 May 2018 Available Online: 10 June 2018}

Klebsiella pneumoniae is a common organism causing bronchopneumonia and is a known pathogen in non-healing diabetic foot infections. KPC strains of Klebsiella pneumonia from Pus and Sputum samples were isolated in Thanjavur Medical College from March to April 2018 which show resistance to beta lactam antibiotics, beta lactamase inhibitors and Carbapenems and confirmed the responsible blaKPC gene by PCR. The study signifies the importance of rational usage of antibiotics.

\section{Introduction}

Klebsiella pneumoniae belongs to the 5th tribe (klebsiellae) among the 8 tribes of Ewing's classification of enterobacteriacae. This nonmotile, indole negative, urease, citrate positive, capsulated, gram negative bacilli is known to cause multiple infections such as bronchopneumonia, sepsis, diabetic foot infections and is one among the 7 nosocomial infections causing organisms (ESKAPES Enterococcus, Staphylococcus aurues, Klebsiella, Acinetobacter, Pseudomonas, Enterobacter, Stenotrophomonas). Widespread and irrational use of antibiotics has caused resistance among Klebsiella pneumoniae to many antibiotics. In this prospective study, we have isolated Klebsiella pneumoniae from pus and sputum samples sent to Department of Microbiology, Thanjavur Medical College and demonstrated various resistance patterns such as ESBL, ampc and KPC.

\section{Materials and Methods}

Identification of Antimicrobial resistance by disc diffusion method using standard discs and confirmation by ertapenem - ertapenem boronic acid MIC strip. Molecular characterization of the confirmed organism was done by PCR. During the study period (March-April 2018), total sample size for pus and sputum were 445 and 232 respectively. Among the 445 pus samples, 39 Klebsiella pneumoniae were isolated and among the 232 sputum samples, 48 Klebsiella pneumoniae 
were isolated. They were screened for ESBL, ampc and KPC.

\section{ESBL detection}

The test organism is streaked by lawn culture method on Muller Hinton agar plate. Ceftazidime (30ug) and ceftazidime clauvalinic acid (30/10ug) standard discs (HiMedia) are kept on the plate. A $5 \mathrm{~mm}$ increase in zone size of ceftazidime clauvalinic acid disc than the ceftazidime disc indicates ESBL strain which implies resistance to betalactams but sensitive to beta lactamase inhibitors.

\section{Ampc detection}

By lawn culture method, on MHA plate, the test organism was streaked and cefoxitin (30ug) and cefoxitin-cloxacillin (30/200ug) standard discs were kept. Ampc producing organisms are resistant to cefoxitin disc and sensitive to cefoxitin-cloxacillin disc which implies, such organisms are resistant to both beta lactams as well as beta lactamase inhibitors.

\section{KPC detection}

KPC (Carbapenamase producing Klebsiella pneumoniae) strains are the emerging superbugs which are resistant to betalactams, beta lactamase inhibitors and Carbapenems.

\section{KPC confirmation by MIC strip}

The test organism is inoculated on MHA plate using lawn culture method, and easy MIC strip having ertapenem on one side and ertapenem boronic acid on the other side is kept. KPC strains show no zone over the side having ertapenem but show zone of inhibition over the side having ertapenem - boronic acid.

\section{Results and Discussion}

Out of the 2 KPC strains isolated from sputum, 1 was from a 30 year old male with a diagnosis of Necrotising pneumonia and the other was from a 71 year old man with a diagnosis of Bronchopneumonia admitted in ICU. KPC isolated from pus sample was that of a 63 year old man's diabetic foot.

The isolated strains were subjected to molecular characterisation by PCR as follows

Bacterial DNA purification was done

The above mentioned components were placed into PCR machine and the following steps were done.

Initial denaturation: $95^{\circ} \mathrm{C}$ for $5 \mathrm{~min}$

Denaturation: $94^{\circ} \mathrm{C}$ for 30 seconds

Annealing: $58^{\circ} \mathrm{C}$ for 30 seconds

Extension: $72^{\circ} \mathrm{C}$ for 30 seconds ( 35 cycles)

Final extension: $72^{\circ} \mathrm{C}$ for $5 \mathrm{~min}$

Fig.1 ESBL strain with difference in zone size of $5 \mathrm{~mm}$ for ceftazidime-clauvalinic acid disc

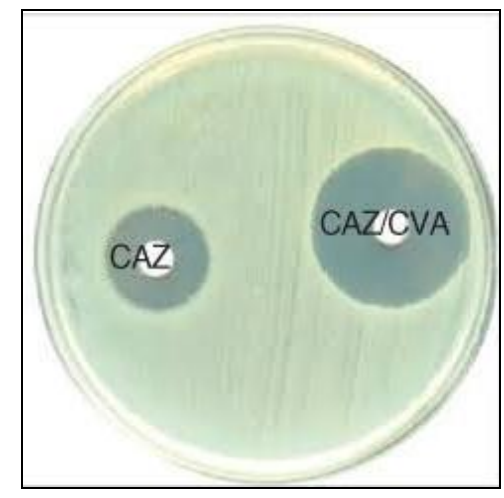


Fig.2 KPC strain showing resistance to ceftazidime, ceftazidime clauvalinic acid, Meropenam and ertapenam and sensitive to colistin

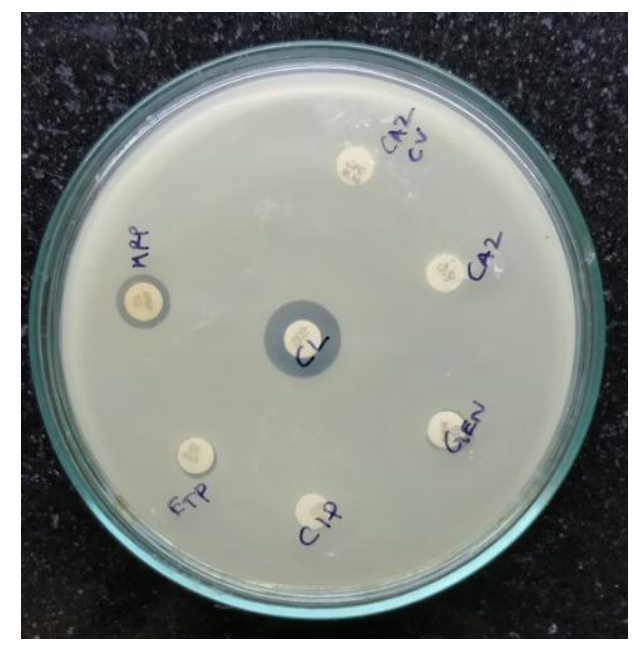

Fig.3 KPC strain with no zone over ertapenem and zone of inhibition over ertapenem-boronic acid

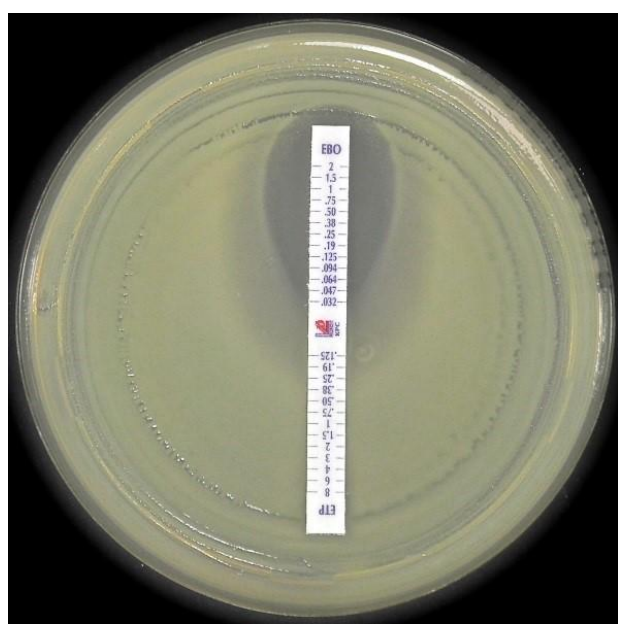

Fig.4 Gel electrophoresis band pattern viewed in UV after PCR

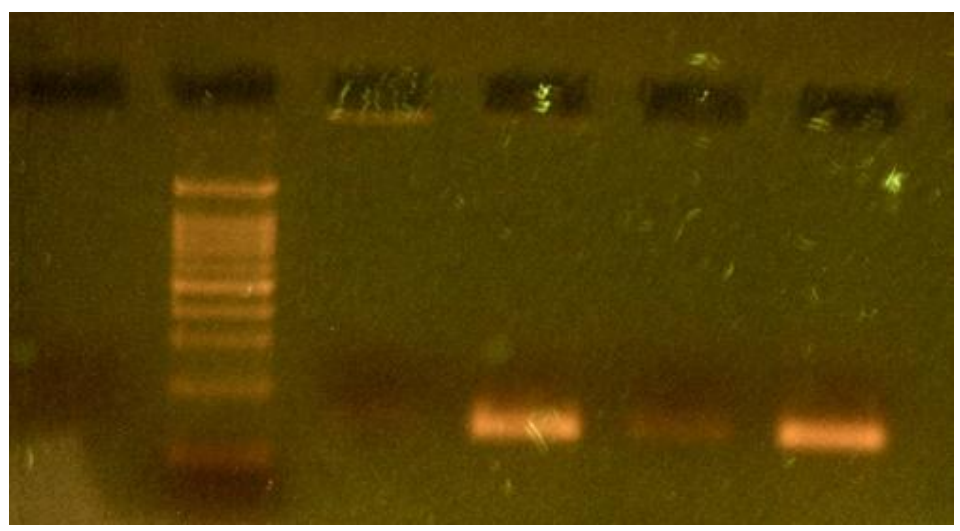




\section{Ambler Classification of $\beta$-lactamases}

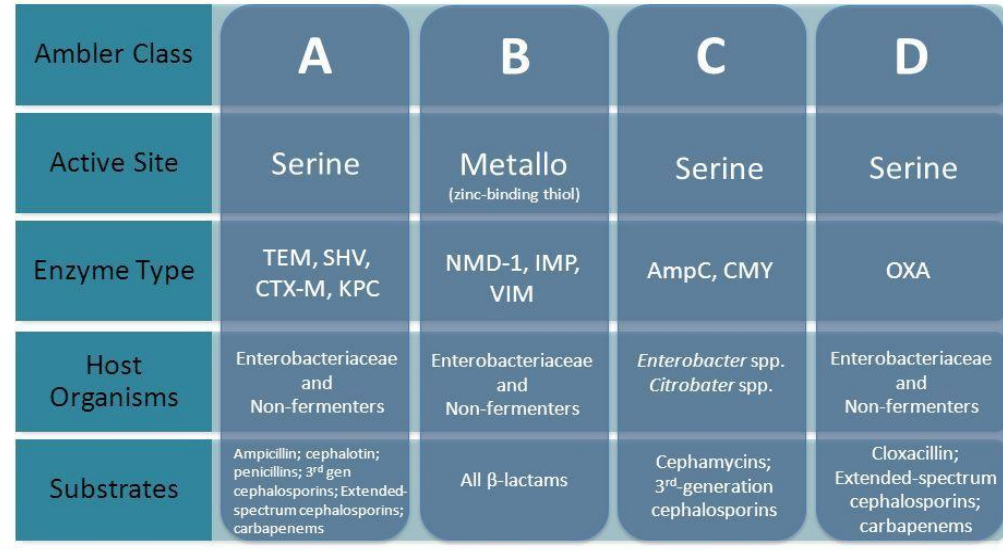

KPC-2 is the most prevalent class A carbapenemase in the world and can hydrolyze the $\beta$-lactamase inhibitors clavulanic acid, sulbactam, and tazobactam.

Table.1 Distribution of ESBL, Ampc, KPC Klebsiella pneumoniae during the study period (Mar-Apr 2018)

\begin{tabular}{|l|l|l|l|l|l|}
\hline Specimen & Total samples & Klebsiella pneumoniae & ESBL & Ampc & KPC \\
\hline Sputum & 232 & 48 & 10 & 6 & 2 \\
\hline Pus & 445 & 39 & 8 & 6 & 1 \\
\hline
\end{tabular}

Table.2 Antimicrobial sensitivity pattern of the $3 \mathrm{KPC}$ strains isolated.

S - Sensitive, R - Resistant

\begin{tabular}{|l|l|l|l|l|l|l|l|}
\hline Diagnosis & Gentamycin & Ciprofloxacin & Ceftazidime & $\begin{array}{l}\text { Ceftazidime- } \\
\text { clauvalinic } \\
\text { acid }\end{array}$ & Ertapenem & Meropenem & Colistin \\
\hline $\begin{array}{l}\text { Necrotising } \\
\text { pneumonia }\end{array}$ & R (14mm) & R (no zone) & R(no zone) & R(no zone) & R(12mm) & R(15mm) & $\begin{array}{l}\mathrm{S} \\
(17 \mathrm{~mm})\end{array}$ \\
\hline $\begin{array}{l}\text { Bronchopneumonia } \\
\text { Diabetic foot }\end{array}$ & R (no zone) & R (no zone) & R (no zone) & R (no zone) & R (14mm) & R (16 mm) & $\begin{array}{l}\mathrm{S} \\
(16 \mathrm{~mm})\end{array}$ \\
\hline & R (none) & R (no zone) & R (no zone) & R (no zone) & R (no zone) & R (10 mm) & $\begin{array}{l}\mathrm{S}(15 \\
\mathrm{mm})\end{array}$ \\
\hline
\end{tabular}

(Zone size for carbapenam resistance is Ertapenam $<18 \mathrm{~mm}$, Meropenam $<19 \mathrm{~mm}$ as per CLSI 2018)

Bacterial DNA purification was done

\begin{tabular}{|l|l|}
\hline Components & Quantity \\
\hline HELINI RedDye PCR Master mix & $10 \mathrm{ul}$ \\
\hline HELINI Ready to use Primer mix & $5 \mathrm{ul}$ \\
\hline Purified Bacterial DNA & $5 \mathrm{ul}$ \\
\hline Total volume & $20 \mathrm{ul}$ \\
\hline
\end{tabular}


Agarose gel electrophoresis was performed and it was viewed in UV transilluminator and the bands were observed.

KPC belongs to class A (serine) of Ambler's classification of beta lactamase. It's a plasmid mediated resistance mechanism. KPC an emerging superbug is a real threat to mankind. The patient with necrotising pneumonia from whose sample KPC was isolated died on the same day of sample collection.

This study isolated Klebsiella pneumoniae from sputum and pus specimens and showed their resistance pattern such as ESBL, ampc and emergence of KPC with a staggering
$3.4 \%$ and emphasises the importance of rational use of antibiotics and proper septic methods to avoid nosocomial infections.

\section{References}

CLSI 2018, page 30 http://journals.plos.org/plosone/article?id=10. 1371/journal.pone.0116421 https://www.sciencedirect.com/science/article /pii/S2210909916301229

Jawets textbook of Microbiology, 27th edition, page. 236

Koneman's colour atlas and textbook of Diagnostic Microbiology edition 7, page. 270

\section{How to cite this article:}

Prakash Murthy E., Eunice Swarna Jacob, B. Hari Prasanth and Ayisha. 2018. Identification of blaKPC Gene from Carbapenemase Producing Klebsiella pneumoniae in Thanjavur Medical College. Int.J.Curr.Microbiol.App.Sci. 7(06): 2613-2617. doi: https://doi.org/10.20546/ijcmas.2018.706.309 